\section{Outstanding Problems in Photometry}

Dr. J. W. T. WALSH gave a thoughtful address on "Outstanding Problems in Photometry" to the Illuminating Engineering Society on February 4. He pointed out that the advent of the gaseous discharge lamp has made the problem of heterochromatic photometry a very urgent one. It is well known that if two surfaces are judged to be equally bright by a number of observers with normal colour vision when the brightness is one candle per square foot, they certainly do not necessarily appear equally bright when the energy they emit is halved (Purkinje effect). Another class of photometric measurements which presents new and difficult problems is the photometry of projection apparatus, such as motorcar headlights, signal lights and similar devices. For such apparatus the conception of candle-power often becomes meaningless. The different optical elements produce beams for which the effective light centres are at such widely separated positions that there is no point that can be approximately regarded as the effective light centre of the whole. Dr. Walsh sug. gests two methods of attack. The first is to abandon candle-power measurements altogether and give a figure or figures for the illumination produced at a specified distance from the face of the projector. The other is to make measurements of candle-power at very great distances from the projector. In the case of a railway signal, this distance may be half a mile. This calls for the use of a telephotometer. A sensitive instrument of this type will probably in the future become part of the regular equipment of any photometric laboratory which has to deal with projection apparatus. In addition, problems arise in deciding what type of measurement is most appropriate in particular cases.

\section{Solar Research at the Kodaikanal Observatory}

The presidential address to the Section of Mathe. matics and Physics at the twenty-third Indian Science Congress was delivered by Dr. T. Royds, director of the Kodaikanal Solar Physics Observatory, who took as his subject "Some Solar Problems" (Calcutta : Asiatic Society of Bengal). Dr. Royds explained that he was dealing with topics which were the subject of present research at Kodaikanal. The first of these was the relation between the dark markings, seen on spectroheliograms, and the prominences. The motion of the dark markings near the limb demon. strates that they are situated at such a height above the photosphere that they would project $31^{\prime \prime}$ above the limb, whereas the average height of prominences is known to be $36^{\prime \prime}$. So far as Kodaikanal observations go, the dark markings and prominences appear to be different aspects of the same thing, that is, a narrow line of flame extending above the sun's surface, having a width of 7,000 miles, a height of 14,000 miles, and an enormous length amounting sometimes to 400,000 miles. Dr. Royds went on to deal with the problem of the support of the chromosphere : it has recently been found at Kodaikanal as the result of observations of the infra-red lines near $7770 \mathrm{~A}$. that oxygen is a normal constituent of the chromosphere. This fact adds to the difficulties in the path of the theory that selective radiation pressure supports the chromosphere. Dr. Royds concluded his address by giving an account of the theory of the intensities of Fraun. hofer lines and of recent measurements which have been carried out at Kodaikanal.

\section{Garden Allotments}

UNTIL the beginning of this century, allotments were chiefly to be found in rural districts; but at the present time, largely owing to the stimulus given to the movement during the Great War, nearly four times the number are situated in urban, as compared with rural, areas. Advice on their management is frequently required, particularly by residents in towns and cities, and the publication by the Ministry of Agriculture of an illustrated bulletin (No. 90. "Allotments". London : H.M. Stationery Office, 1s.) should prove of immense practical value to all holding or contemplating holding an allotment. The scope of the bulletin is wide, and only an indication of its range can be given here. Questions of soils and their treatment, manures and manuring, with a special section on lime, are dealt with in some detail. Full directions follow for the cultivation of all the ordinary market garden crops, such as potatoes, the cabbage group, peas, beans and root vegetables and to a lesser extent fruit and flowers. Advice is also given on simple methods for purposes of protection and forcing. Only a small section is devoted to pests and diseases, as the reader is referred to the more detailed publications on the subject already issued by the Ministry. At the end of the bulletin a summary of work appropriate for each month of the year will be found, together with useful hints for those intending to exhibit their vegetables at shows. Further, the business side of allotment holding is not overlooked, and a part of the bulletin is devoted to questions of rules for allotment associations, tenancy agreemonts, choice, planning and layout of sites and the methods by which an individual can, where practicable, acquire an allotment.

\section{National Institute of Agricultural Botany}

THE National Institute of Agricultural Botany has just published its sixteenth report, which records the services provided in 1935 by the Crop Improvement Branch and the Official Seed Testing Station at Cambridge and the Potato Testing Station at Orms. kirk. A small quantity of the new variety of wheat 'Holdfast' was marketed during 1935. This wheat was bred from a cross between 'Yeoman' and 'White Fife', has a white chaff and white grain and shows a marked resistance to 'lodging'. It is particularly well fitted for growing on good land in high condition. The number of samples tested by the Official Seed Testing Station was 30,502, the highest on record. The work of the Potato Testing Station was seriously hampered by the abnormal weather conditions, but the usual tests of new varieties for susceptibility to wart disease were carried out, together with a number of yield and quality trials. A Lord Derby Gold Medal was awarded to 'Gladstone', a new potato produced 